\title{
Simple model for the phase coexistence and electrical conductivity of alkali fluids
}

\author{
P. Tarazona ${ }^{1}$, E. Chacón ${ }^{2}$, and J. P. Hernandez ${ }^{3}$ \\ ${ }^{1}$ Departamento de Física de la Materia Condensada (C-XII), Universidad Autónoma de Madrid, \\ E-28049 Madrid, Spain \\ ${ }^{2}$ Instituto de Ciencia de Materiales, Consejo Superior de Investigaciones Científicas and \\ Departamento de Física Fundamental, Universidad Nacional de Educación a Distancia, Apartado \\ 60141, E-28028 Madrid, Spain \\ ${ }^{3}$ Department of Physics and Astronomy, University of North Carolina, Chapel Hill NC \\ 27599-3255 USA
}

(August 3, 2018)

\begin{abstract}
We report the first theoretical model for the alkali fluids which yields a liquid-vapor phase coexistence with the experimentally observed features and electrical conductivity estimates which are also in accord with observations. We have carried out a Monte Carlo simulation for a lattice gas model which allows an integrated study of the structural, thermodynamic, and electronic properties of metal-atom fluids. Although such a technique is applicable to both metallic and nonmetallic fluids, non-additive interactions due to valence electron delocalization are a crucial feature of the present model.
\end{abstract}

PACS numbers: 61.25.Mv, 64.70.Fx, 71.30.+h 
Until recently little has been known regarding metal-atom fluids near the high temperatures and pressures characteristic of their liquid-vapor critical points. However, such knowledge is not only of considerable scientific interest but is also required for potential technological applications near those conditions. The electronic and thermodynamic properties of such fluids are intimately related and knowledge of these properties is essential in determining the right material for a given application. Such considerations have motivated experimental studies of those materials with the lowest critical temperatures: $\mathrm{Hg}(1751 \mathrm{~K})$, Cs (1924 K), and Rb (2017 K) [1], with data on K (2178 K) now being available [2]. The data have become precise and reliable in the last decade and span thermodynamic and electrical measurements under the same conditions. Such data show that the liquid-vapor coexistence curve of metal-atom fluids are different from those of Lennard-Jones-like ones [2, 3] . For example, the liquid and vapor branches of the coexistence curves are strongly asymmetric and the rectilinear diameter law breaks down over a large temperature range, not only very close to the critical points. These materials also undergo a metal-nonmetal (M-nM) transition. This body of data, however, still seeks microscopic theoretical foundations [4]. This paper presents a simple model which appears to contain the basic ideas required to reproduce, in a unified manner, the peculiar characteristics observed in the alkali fluids.

The goal of the present work - understanding the structural, thermodynamic, and electronic properties of metal-atom fluids - poses a considerable scientific challenge. Its various aspects are coupled since it is the electrons which determine interatomic interactions and thus the structure and thermodynamic data. The ionic structure, in turn, determines the electronic properties. In the study of metal-atom fluids it is difficult to impose a structure, since it is so intimately related to electronic effects and there is no long-range symmetry to simplify the problem. Also, because in such materials the interactions are not pairwise ones for intermediate densities, due to electron delocalization over some regions, the problem is more complicated than that for fluids in which the interactions are not state dependent. Similarly, as these materials also undergo a M-nM transition, the traditional techniques used to study free-electron-like fluids are not applicable over many of the conditions of interest. 
The microscopic theory required for these materials should seek to explain the essential interdependence of thermodynamic, structural, and electronic properties. Previous theoretical efforts to comprehensively explain the available experimental data on metal-atom fluids have been sparse. The points of view taken were usually based on the limiting cases of either a metallic dense liquid or solid, or alternatively of a nonmetallic dilute vapor. Attempts were then made to describe the fluid, or some of its properties, in a limited density and temperature range [5]. General arguments based on electron correlation effects (Hubbard model) and/or disorder induced localization (Anderson model) [8] are useful to study the M-nM transition in systems with frozen ionic structure but probably not in metal-atom fluids.

A first step towards a comprehensive theoretical treatment attempted the extension of concepts and techniques of plasma physics to treat both liquid and vapor phases, and then tried to introduce the required neutral atoms and small clusters [9]. We also proposed such an approach [10,11] and showed that a toy model gives a liquid-vapor coexistence and a critical point with some correct features, but we were far from reproducing the peculiar coexistence curve of alkali fluids. In recent work [12], we followed this approach including a quantitatively good description of the plasma: a standard description of liquid metals near their triple point [13]. Extension of this treatment to high temperature and low density gave a plasma liquid-vapor coexistence with a very high critical temperature (around four times the experimental value) and a very different shape for the coexistence curve than that observed. We then extended the model to allow for chemical coexistence of neutral atoms with the plasma. Virial ion-atom interactions were used, instead of their neglect as in the previous toy model. However, reasonable values of the parameters did not improve the previous plasma results. We have concluded that a mean-field theory is not capable of reproducing the structure or phase diagram of the alkali fluids [12].

An unanswered question, in the above approach, is: why do some fraction of the valence electrons choose to be bound in atoms while others are delocalized, at fixed temperature and density? The answer must lie in a hitherto ignored underlying structure. An important clue is that clustering effects are strongly enhanced for metal-atom systems, compared to 
nonmetallic ones, due to their high cohesion which arises from the valence electron delocalization over the cluster. In contrast, to retain its valence electron an atom should have no near neighbors to which that electron can be favorably delocalized. We present here a simple lattice gas model which includes such considerations and yields results showing the observed peculiarities of the alkali fluids.

In our model, we allow the ions to partially occupy the sites of a body centered cubic (bcc) lattice, which has the same maximum number of nearest neighbors as do real metals. The lattice parameter is determined by the condition that the maximum density $\rho_{0}$ be equal to that of the liquid metal at its triple point. The energy, in a mean-field treatment of the model, is obtained from the energy per ion $u(\rho)$ in a normal treatment for a liquid metal with mean density $\rho$ [13]. That is, using a jellium reference system for the delocalized valence electrons yields kinetic, exchange, and correlation energy contributions, and a linear response function. Also, a hard-sphere reference for the ions gives a pair distribution function. Finally, a pseudopotential is associated with the ions (Ashcroft, empty core, fitted to the liquid metal conductivity at the melting point) and the screened ion-electron and ionion interactions (minus reference system effects) are treated by perturbation theory. This is a Gibbs-Bogoliubov variational approximation. The electronic effects are treated at zero temperature since no appreciable changes result from including the temperature effects in the kinetic and exchange energies. The results with the mean-field lattice model (Table I) are very close to those of the previous, continuous, plasma model [12.

The crucial point, however, is to calculate the energy of the system taking into account the strong inhomogeneities due to clustering. We now propose to get the energy per ion using the mean-field energy $u(\hat{\rho})$ which is to be evaluated at a local average density $\hat{\rho}$. This density takes into account the ion and its environment, through the occupation of its nearest-neighbor lattice sites. Thus, $\hat{\rho}=(n+1) \rho_{0} / 9$ and $\mathrm{n}$ is the number of occupied nearest-neighbor sites; it takes values from 0 to 8 . For ions without any nearest neighbors $(n=0)$, this energy, calculated as in a plasma, is compared with that of a free atom (i.e. minus the ionization energy ) and in a variational spirit the lower value is chosen. Although 
the energy difference is small, the atomic state is lower for all the alkalis. This approach thus includes the structurally-based possibility of valence electron localization, to form atoms, or some degree of delocalization. For cesium we take $\rho_{0}=1.84 \mathrm{~g} / \mathrm{cm}^{3}$ and obtain the following energies per ion (in eV):

$\begin{array}{llllllllll}n & 0 & 1 & 2 & 3 & 4 & 5 & 6 & 7 & 8\end{array}$

$u-3.89-4.20-4.42-4.58-4.71-4.81-4.90-4.99-5.06$.

This simple model for the configuration energy contains a basic difference with the pairwise interactions of usual lattice models: non-additive interactions with neighboring ions due to valence electron delocalization. Also, such interactions are misrepresented in a mean-field treatment of a metal-atom fluid if the density fluctuations become important.

We present the results of Monte Carlo simulations for this model, applied to cesium and potassium. We have carried out simulations within a cube with twelve bcc cells on each side (3456 sites). Some results have been checked using a larger cube (8192 sites), without appreciable changes in the results. The simulations, carried out at fixed temperature and chemical potential, give the equilibrium density and internal energy of the system. The pressure is obtained by thermodynamic integration. Structural features such as a nearly linear decrease in the average coordination of ions in the expanded coexisting liquid result from this calculation; that feature agrees with what has been deduced from analysis of neutron scattering data [14]. In Fig. 1, we show the coexistence curves, in critical reduced units, obtained for cesium along with the experimental result. Using the present method, the shape of the coexistence curve is in good agreement with experiments, recovering the strong liquid-vapor asymmetry. Although the size of our simulation box prevents a closer approach to the critical point than about one per cent, our results clearly show a non-classical critical behavior, in agreement with experiment and previous theoretical predictions [15],16]. Near the critical point, the diameter $\rho_{d}=\left(\rho_{L}+\rho_{V}\right) / 2 \rho_{C}$ is indicated in the figure and clearly shows the nonlinear behavior: $\rho_{d}-1 \propto\left|\left(T_{C}-T\right) / T_{C}\right|^{1-\alpha}$. Our estimate for $1-\alpha, 0.8 \pm 0.1$, is compatible with the experimental value [3] of $0.87 \pm 0.03$. We have also calculated the 
phase diagram for potassium; in reduced critical units it is very similar to that of cesium. In agreement with experiments, our results for different alkali fluids give similar reduced phase diagrams.

The critical temperatures in our model are higher than those of the real fluids, as one would expect from a lattice-gas treatment of the configurational entropy. However, the results in table I show that the correlation effects included in the present model produce a very large decrease of the critical temperature compared with the previously mentioned mean-field approach. Also, the critical densities and pressures are brought into reasonable agreement with experiments. Moreover, the relative critical parameters of cesium and potassium in our model are very similar to the relative experimental ones.

We have next explored electronic properties related to structure. The experimental signature of a M-nM transition in these systems is a decrease of several orders of magnitude in the conductivity of the expanded fluid metal. In our model, such behavior is driven by the percolation of the ionic cluster structure, rather than by considerations such as those of Mott or Anderson. From typical configurations of our Monte Carlo simulation for cesium, we have obtained the cluster structure at different temperatures and pressures. The conductivity was estimated following the Kirchoff's law model proposed by Nield et al. [17], with arbitrary units fitting the experimental conductivity at the maximum density. The results in Fig.2 show the conductivity estimated in this manner along the critical isotherm versus the pressure, in excellent agreement with experiment [1]. The figure also shows the calculated conductivity versus density at the critical temperature, and that arising from a random filling of the lattice. The difference clearly shows the effects of clustering. The percolation density (at which the conductivity goes to zero) at $T_{c}$ is less than half that obtained with random occupation.

We have presented a model allowing a unified study of the structural, thermodynamic, and electronic properties of metal-atom fluids. The model takes into account non-additive interactions, due to valence electron delocalization, and it is studied with a Monte Carlo simulation which goes beyond mean-field, as is required. Although the model is a very 
simplified representation of a metal-atom fluid, comparisons with a range of experimental results show that it contains the basic ingredients to allow reproduction of the peculiar behavior observed in these systems. These peculiarities include the metal-nonmetal and liquid-vapor transitions and the connection between ionic and electronic structures. Our results for the critical temperatures are still too high, though the densities and pressures are acceptable. It is reasonable to expect that, with a more realistic description of the fluid entropy, a similar model (though more cumbersome to study) would also give a quantitatively good result for the critical temperatures. Our simplified model appears to allow a unified understanding of the peculiar characteristics of the alkali fluids.

This work was supported by the Dirección General de Investigación Científica y Técnica (Spain) under Grant PB91-0090 and the Instituto Nicolas Cabrera. 


\section{REFERENCES}

[1] F. Hensel in Physics and Chemistry of Electrons and Ions in Condensed Matter, J. V. Acrivos, N. F. Mott, and A. D. Yoffee, eds. (Dordrecht, Reidel, 1984) p. 401; F. Hensel, S. Jungst, F. Noll, and R. Winter in Localization and Insulator-Metal Transitions, D. Adler and H. Fritzche, eds. (New York, Plenum, 1985) p. 109; F. Hensel, H. Uchtmann, Annu. Rev. Phys. Chem. 40, 61 (1989)

[2] G. Hohl, Ph. D. thesis, Marburg 1992 (unpublished)

[3] S. Jüngst, B. Knuth, F. Hensel, Phys. Rev. Lett. 55, 2164 (1985)

[4] R. M. Stratt, Annu. Rev. Phys. Chem. 41, 175 (1990)

[5] From Hamiltonians to Phase Transitions, Springer series in solid-state sciences 70, by J. Hafner (Springer-Verlag, Heidelberg, 1987)

[6] P. Minchin, M. Watanabe, W. H. Young, J. Phys. F: Metal Phys. 7, 563 (1977)

[7] J. P. Hernandez, Phys. Rev. Lett. 57, 3183 (1986)

[8] P. A. Lee, T. V. Ramakrishnan, Rev. Mod. Phys. 57, 287 (1985)

[9] See Quantum Statistics of Charged Particle Systems by W. Kraeft, D. Kremp, W. Ebeling, G. Ropke (Plenum, New York., 1986); also, R. Redmer, G. Ropke, Physica 130A, 523 (1985) and Contrib. Plasma Phys. 29, 343 (1989), R. Redmer and W. W. Warren,Jr., Phys. Rev. B 48, 14892 (1993)

[10] E. Chacón, J. P. Hernandez, P. Tarazona, J. Phys.: Condens. Matter 5, 1753 (1993)

[11] J. P. Hernandez, Z. Phys. Chem. (1994), to be published

[12] P. Tarazona, J. P. Hernandez and E. Chacón, unpublished. A brief report of part of this work was presented at the VII International Workshop on the Physics of Non-Ideal Plasmas (Rostock, 1993); see P. Tarazona, E. Chacon, J. P. Hernandez, Contrib. Plasma Phys. 33, 370 (1993) 
[13] Liquid Metals by M. Shimoji (Academic Press, New York 1977) p. 28; N. W. Ashcroft and D. Stroud in Sol. St. Phys. 33, H. Ehrenreich, F. Seitz and D. Turnbull, eds. (Academic, New York 1978) p. 1; and ref. 5

[14] R. Winter, C. Pilgrim, F. Hensel, C. Morkel, and W. Glaser, J. Non-Cryst. Solids 156158, $9(1993)$

[15] R. E. Goldstein, N. W. Ashcroft, Phys. Rev. Lett. 55, 2164 (1985)

[16] R. E. Goldstein, A. Parola, A. P. Smith, J. Chem. Phys. 91, 1853 (1989)

[17] V. M. Nield, M. A. Howe, R. L. McGreevy, J. Phys.: Condens. Matter 3, 7519 (1991) 


\section{FIGURES}

FIG. 1. Liquid-vapor coexistence curve of cesium. Filled circles: present MC simulation; full line: fit to the experimental results of ref. [3]. The MC simulation diameter function, $\rho_{d}$, is also plotted (crosses). The triangles show our metal-nonmetal transition (i.e. the percolation line), the dotted line is a guide to the eye.

FIG. 2. On the left we show the electrical conductivity $\sigma$ versus the reduced pressure along the critical isotherm: crosses - our model ; open circles - experimental values of ref. [1], both normalized to the conductivity $\sigma_{0}$ at our highest density $\rho_{0}$. On the right we show our calculated electrical conductivity but now versus the fractional occupation of the lattice; crosses are our results for $T=T_{c}$ and triangles for the random filling of the lattice. 


\section{TABLES}

TABLE I. Comparison of the calculated critical conditions, using lattice gas mean-field and the present theory, with the experimental results of ref. [2] and [3]. The temperature $T$ is in Kelvin, the pressure $P$ in bar, and the density $\rho$ in $\mathrm{g} \mathrm{cm}^{-3}$.

\begin{tabular}{|c|c|c|c|c|c|c|}
\hline & \multicolumn{3}{|c|}{ CESIUM } & \multicolumn{3}{|c|}{ POTASSIUM } \\
\hline & $T_{c}$ & $\rho_{c}$ & $P_{c}$ & $T_{c}$ & $\rho_{c}$ & $P_{c}$ \\
\hline Mean Field & 6730 & 0.03 & 9.6 & 7890 & 0.018 & 19 \\
\hline Our Theory & 3600 & 0.50 & 100 & 3920 & 0.21 & 170 \\
\hline Experiment & 1924 & 0.38 & 92.5 & 2178 & 0.18 & 148 \\
\hline
\end{tabular}

